\title{
Workspace Awareness Acessível: estratégias de sonificação para projetar interfaces colaborativas acessíveis aos cegos
}

\author{
Márcio J. R. Torres ${ }^{12}$, Regina Barwäldt ${ }^{1}$ \\ ${ }^{1}$ Centro de Ciências Computacionais (C3) - Universidade Federal do Rio Grande (FURG) \\ Rio Grande - RS - Brasil \\ ${ }^{2}$ Instituto Federal de Educação, Ciência e Tecnologia do Rio Grande do Sul (IFRS) \\ Rio Grande - RS - Brasil \\ marcio.torreseriogrande.ifrs.edu.br, reginabarwaldtefurg.br
}

\begin{abstract}
The extensive use of diagrams in vocational and higher education, especially in computing and engineering courses, is challenging for blind and visually impaired students. Despite advances and studies about the accessibility of diagrammatic information, they do not address blind-friendly collaborative resources. Therefore, this study was carried out to handle this issue by designing two audible interfaces based on sonification resources. These interfaces went through usability and cognitive load tests. The results showed that blind users were able to perceive the interactions of colleagues through sonified interfaces. They could distinguish which action was performed, where it took place and made by whom.
\end{abstract}

Resumo. O uso extensivo de diagramas no ensino profissional e superior, sobretudo nos cursos de computação e engenharias, impõem um obstáculo ao aprendizado de cegos e deficientes visuais e, apesar dos avanços e estudos quanto à acessibilidade da informação diagramática, eles não tratam de recursos colaborativos acessíveis aos cegos. Portanto, foi conduzido um estudo onde foram projetadas duas interfaces audiveis baseadas em recursos de sonificação. As interfaces passaram por testes de usabilidade e carga cognitiva. Os resultados demonstraram que usuários cegos podem perceber as interações dos colegas e distinguir a ação realizada, o local onde aconteceu e quem a realizou por meio das interfaces sonificadas.

\section{Introdução}

Existem políticas públicas para assegurar o acesso e permanência de PcDs nas instituições públicas de ensino, como as leis no. 12.711/2021 e 13.409/2016, que garantem cotas para minorias de qualquer tipo, incluindo Pessoas com Deficiência (PcD), em cursos da rede de educação profissional e ensino superior. As matrículas de PcDs na educação profissional, por exemplo, cresceu de 2.899 em 2010 para 6.206 em 2016 [INEP 2020]. No entanto, ainda existem desafios tais como a falta de ferramentas acessíveis aos cegos e deficientes visuais para o desenho de diagramas.

Diagramas, entre outros recursos gráficos e visuais, são amplamente empregados no campo da educação em ciências e engenharias [Cryer et al. 2013], sobretudo na computação, tecnologias da informação e áreas correlatas. Diversos estudos relataram dificuldades no ensino de tópicos de engenharia que se apoiam nos 
recursos diagramáticos para transmitir conceitos, por exemplo, modelagem UML [Luque et al. 2014, Müller 2012], estruturas moleculares [Brown et al. 2004a] e gráficos estatísticos [Godfrey et al. 2018], entre outros. Existem estudos sobre a acessibilidade de diagramas, dedicados à percepção [King et al. 2004] e autoria [Metatla et al. 2008], no entanto, há poucos estudos sobre a colaboratividade [Torres and Barwaldt 2019].

O workspace awareness (conscientização ou ciência do espaço de trabalho), é o subcampo específico dentro de groupware que estuda o modo como as pessoas percebem a interação dos outros dentro de um ambiente multiusuário compartilhado. Aplicativos de groupware inclusivos devem providenciar um workspace awareness acessível, permitindo que usuários cegos estejam conscientes das ações dos colegas usando outra estratégia de notificação que não as baseadas em recursos gráficos e visuais [Torres et al. 2020].

Portanto, o objetivo deste estudo foi de projetar e avaliar a sonificação dos eventos resultantes de interações nas interfaces compartilhadas. Para tornar perceptíveis e discerníveis aos usuários cegos, foram projetadas estratégias para o mapeamento dos elementos de workspace awareness a pistas audíveis reproduzidas por sons abstratos chamados Earcons para sinalizar três eventos em três categorias: (a) quem está fazendo? (b) o que está fazendo? e (c) onde está fazendo?

\section{Referencial Teórico}

Para ser acessível aos cegos, podem ser usados recursos audíveis ou hápticos para representar as informações. Nas possibilidades audíveis, existem opções verbais e nãoverbais, isto é, soluções baseadas na fala ou em sons não reconhecíveis como um discurso. Este trabalho, especificamente, aplica técnicas não-verbais de sonificação para o projeto e implementação do workspace awareness, detalhados nos tópicos a seguir.

\subsection{Sonificação}

A sonificação é a transformação de dados relacionados em sinais audíveis que podem ser comunicados e interpretados, onde os sons não são reconhecíveis como um discurso [Hermann 2008]. Existem diversas técnicas de sonificação para converter e representar informação para e em áudio. No entanto, as duas mais conhecidas e comuns são as opções de ícones auditivos e Earcons.

Os ícones auditivos têm a intenção de imitar os sons do mundo-real. Eles são para sons o que ícones são para imagens, supondo que os usuários captem o significado da mesma forma que suas contrapartidas visuais, por meio do relacionamento entre um significado e significante[Gaver 1986].

Os Earcons, por outro lado, são mensagens na forma de sons distintos o suficiente para especificar eventos e transmitir informações [Blattner et al. 1989]. Por não ter correspondência direta com o mundo-real, os Earcons dependem do projeto acústico para transmitir e comunicar diferentes partes de informação por meio de, por exemplo, a variação do timbre, altura, frequência, intensidade e amplitude do sinal audível [Blattner et al. 1989].

\subsection{Workspace Awareness}

O Workspace Awareness (WA) é descrito como "a coleção minuto-a-minuto do conhecimento que uma pessoa dispõe sobre o estado das interações dos outros no workspace." 
[Gutwin and Greenberg 1996]. O framework conceitual desenvolvido por Gutwin e Greenberg trata dos desafios comuns no projeto de groupware relacionados às informações que devem ser coletadas e apresentadas em sistemas distribuídos em tempo-real. Estas informações foram classificadas em categorias e elementos que devem responder às seguintes questões elencadas na Tabela 1, a seguir:

Tabela 1. Elementos de Workspace Awareness [Gutwin and Greenberg 2002].

\begin{tabular}{c|c|l}
\hline Categoria & Elemento & Questões específicas \\
\hline \multirow{4}{*}{ Quem } & Presença & Há alguém no workspace? \\
\cline { 2 - 3 } & Identidade & Quem está participando? Quem é esse? \\
\cline { 2 - 3 } O quê/Qual & Autoria & Quem está fazendo isso? \\
\cline { 2 - 3 } & Ação & O que estão fazendo? \\
\cline { 2 - 3 } & Intenção & Essa ação tem qual objetivo? \\
\hline \multirow{4}{*}{ Onde } & Artefato & Em que objeto estão trabalhando? \\
\cline { 2 - 3 } & Localização & Onde estão trabalhando? \\
\cline { 2 - 3 } & Olhar & Para onde estão olhando? \\
\cline { 2 - 3 } & Visão & Até onde podem ver? \\
\cline { 2 - 3 } Como & Alcance & Até onde podem alcançar? \\
\cline { 2 - 3 } Quando & Histórico de Ações & Como isso aconteceu? \\
\cline { 2 - 3 } & História do Artefato & Como acabou nesse estado? \\
\hline
\end{tabular}

\section{Metodologia}

Este estudo foi conduzido durante dois anos, desde a revisão da literatura [Torres and Barwaldt 2019] até o projeto da sonificação e implementação em um protótipo que foi, então, submetido a testes de usabilidade e carga cognitiva.

\subsection{Teste de Usabilidade: Taxa de Sucesso}

Os testes de usabilidade, ou testes com usuários, são métodos empíricos para avaliar uma solução na área de Interação Humano-Computador (IHC). Podem ser coletados dados qualitativos ou quantitativos, enquanto o primeiro foca na revelação dos comportamentos dos usuários enquanto usam o produto, o segundo coleciona métricas que descrevem a experiência objetiva do usuário, tais como a taxa de sucesso e o tempo despendido na tarefa [Nielsen 2001]..

Nesta pesquisa, especificamente, notou-se os efeitos da baixa representação populacional durante o recrutamento de sujeitos para o teste, condição que também foi observada em outros estudos com cegos e deficientes visuais [Thapa et al. 2017, Freire et al. 2010]. Contudo, mesmo os testes de usabilidade com cinco usuários podem revelar boa parte dos problemas [Turner et al. 2006, Nielsen 2012].

\subsection{Medição de Carga de Trabalho: NASA-TLX}

A ferramenta NASA Task Load Index (NASA-TLX) é usada para mensurar a carga de trabalho que representa o custo para um usuário completar as exigências de uma tarefa e busca quantificar o esforço realizado por um indivíduo para identificar fadiga, stress e outros fatores que podem mitigar o desempenho [Hart 1986]. Ela possui duas partes: (1) a separação em seis dimensões, ou subescalas; (2) definição de pesos individuais para cada subescala. Quando a segunda parte é suprimida, o exame é conhecido como unweighted, ou NASA Raw Task Load Index (NASA-RTLX). As dimensões são: demanda mental, demanda física, demanda temporal, performance, esforço e frustração. Para cada dimensão 
é atribuído um valor de 0 a 100 com saltos de 5 pontos. Na versão unweighted ou raw, o score final é obtido por meio da média dos valores para cada subescala e arredondando para o valor mais próximo da escala (0 a 100 em intervalos de 5), o qual foi utilizado neste estudo.

\section{Workspace Awareness Acessível}

Este estudo propõe que os usuários cegos possam perceber as ações dos outros usuários por meio de uma interface de usuário audível baseada em técnicas de sonificação onde os elementos essenciais de workspace awareness são mapeados para pistas sonoras. Para projetar esta interface, seguiu-se o framework conceitual de Gutwin e Greenberg, destacando um conjunto básico de elementos nas três categorias de workspace awareness relativas ao presente: "Quem”, "O quê" e "Onde".

Neste processo, foram projetadas duas interfaces audíveis para a modelagem colaborativa de diagramas, baseadas em técnicas de sonificação com Earcons para comunicar quatro informações pontuais em um workspace compartilhado (1) Autoria/Quem (quem é o responsável por essa ação) (2) Ação/O quê (o quê foi feito?) (3) Localização/Direção (em quê direção essa atualização aconteceu) e (4) Localização/Distância (quão distante aconteceu esta atualização). Cada peça de informação foi codificada usando recursos audíveis como apresentado nos tópicos a seguir.

\subsection{Sonificação das Interfaces}

Foram projetadas duas interfaces sonificadas, chamadas de SONIv1 e SONIv2, com variações nas estratégias para comunicar informações nas três categorias: autoria, ação e localização.

A categoria autoria responde a pergunta "quem fez a ação?”. Ambas interfaces SONIv1 e SONIv2 foram inspiradas em como a escrita colaborativa online funciona, representando cada usuário por um cursor e avatar colorido. Logo, o projeto do Earcon para a pista audível foi baseado na "cor do tom", conhecido como timbre, que permite a diferenciação de sons com a mesma frequência (nota e altura) através de instrumentos musicais distintos. Logo, sons reproduzidos por diferentes instrumentos são análogos à cores diferentes.

A categoria ação responde a pergunta "o que foi feito?”. A sonificação foi projetada para reproduzir espaçadamente as notas variando de forma contínua (sem variação de altura), ascendente (das notas baixas às altas) ou descendente (das notas altas às baixas), para representar respectivamente alteração, inclusão e exclusão. Por exemplo, o ato de incluir um artefato pode ser representado pela reprodução do acorde "Dó maior" de modo que as notas Dó-Mi-Só são reproduzidas sequencialmente em vez de simultaneamente, enquanto a reprodução de $S o ́-M i$-Dó sequencialmente, representaria a sua exclusão, conforme ilustração na Figura 1. Ademais, as interfaces SONIv1 e SONIv2 diferiram um pouco a estratégia, sendo que a interface SONIv1 foi projetada para reproduzir apenas duas notas e a SONIv2 foi projetada para reproduzir uma quantidade proporcional a distância.

A categoria localização responde a pergunta “onde ocorreu a ação?”. Esta categoria foi desmembrada em duas sinalizações distintas: a (1) direção relativa (esquerda, frente ou direita) e (2) a distância. 
Figura 1. Sketch do efeito sonoro para o awareness de "o quê" (fonte: autor).

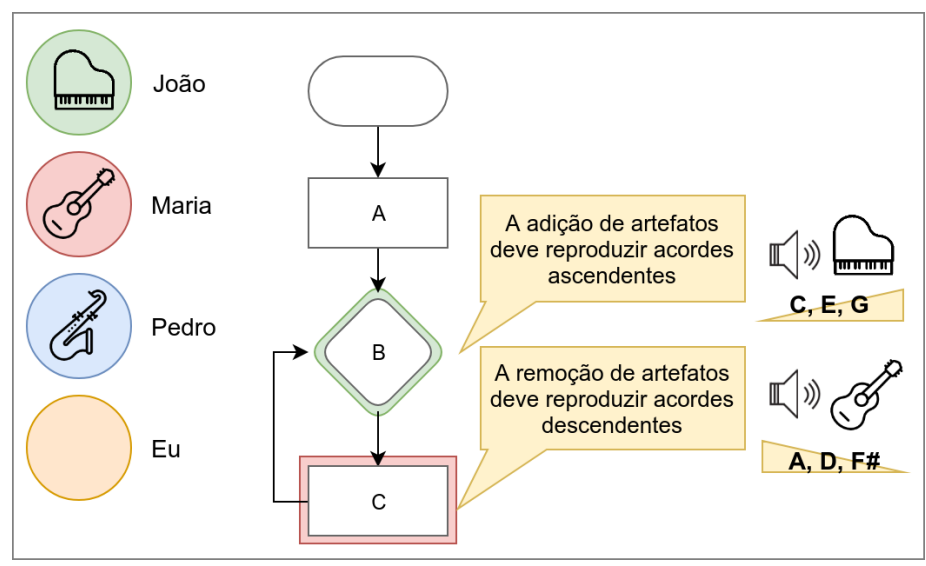

Ambas interfaces SONIv1 e SONIv2 foram projetadas para transmitir a localização/direção por meio do efeito estereofônico, isto é, as pistas audíveis são reproduzidas no fone de ouvido de acordo com a direção relativa nos canais esquerdo ou direito, ou ambos canais no caso de intervenções em frente ao usuário (o artefato/vértice selecionado pelo usuário cego). Alguns autores consideram relevante a posição bidimensional dos objetos no espaço. Soluções que apoiam-se no espaço $2 \mathrm{D}$, tipicamente comunicam as coordenadas por meio de recursos cinestético [Brown et al. 2004b]. Aqueles estudos que propõem transmitir a posição apenas por meio de recursos auditivos tipicamente o realizam por meio de som espacializado [Miura et al. 2016, Balan et al. 2015] ou combinando recursos hápticos e audíveis [Syal et al. 2016, Cohen et al. 2006] (multimodal). Neste estudo, no entanto, é proposto um leiaute espacial substituto ao modelo bidimensional, baseado apenas nestas três direções relativas, que permite comunicar o posicionamento por meio de fones de ouvido estéreo convencionais.

Quanto à localização/distância, foram empregados dois efeitos sonoros, um para cada interface projetada, SONIv1 e SONIv2. Na SONIv1 foi projetado o desvanecimento do volume, ou efeito de fade, que consiste na variação da intensidade sonora para sinalizar a distância. Foram definidos quatro níveis de intensidade, conforme pode ser visto na Figura 2). O efeito de fade foi projetado para reduzir o volume em $40 \%$ em relação ao nível anterior, resultando em quatro níveis: (1) 100\% de volume (para ações no artefato selecionado), (2) 60\% de volume (para ações adjacentes em até dois links de distância), (3) $24 \%$ de volume (ações próximas em artefatos até quatro links de distância) e (4) $10 \%$ de volume (ações distantes, 5+ links de distância).

A segunda interface, SONIv2, foi projetada para sinalizar a distância por meio da variação da quantidade de notas musicais e da intensidade durante a reprodução. Foram projetados seis níveis, reproduzidos com as notas Dó-Ré-Mi-Fá-Sol-Lá-Si em altura ascendente nas inclusões, descendente ( $\mathrm{Si}-\mathrm{Lá}$-Sol ...) nas exclusões, e constante ( $n$ l inks + 1 vezes a nota Dó) nas alterações. Nesta estratégia, a intensidade acompanha a altura das notas em partes iguais, o que produz os efeitos de fade-in e fade-out nas inclusões e exclusões, respectivamente, conforme apresenta a Figura 2. No caso das alterações, a nota Dó é reproduzida "distância +1 " vezes com volume constante. 
Figura 2. Sketch da sonificação da distância em SONIv1 e SONIv2 (fonte: autor).

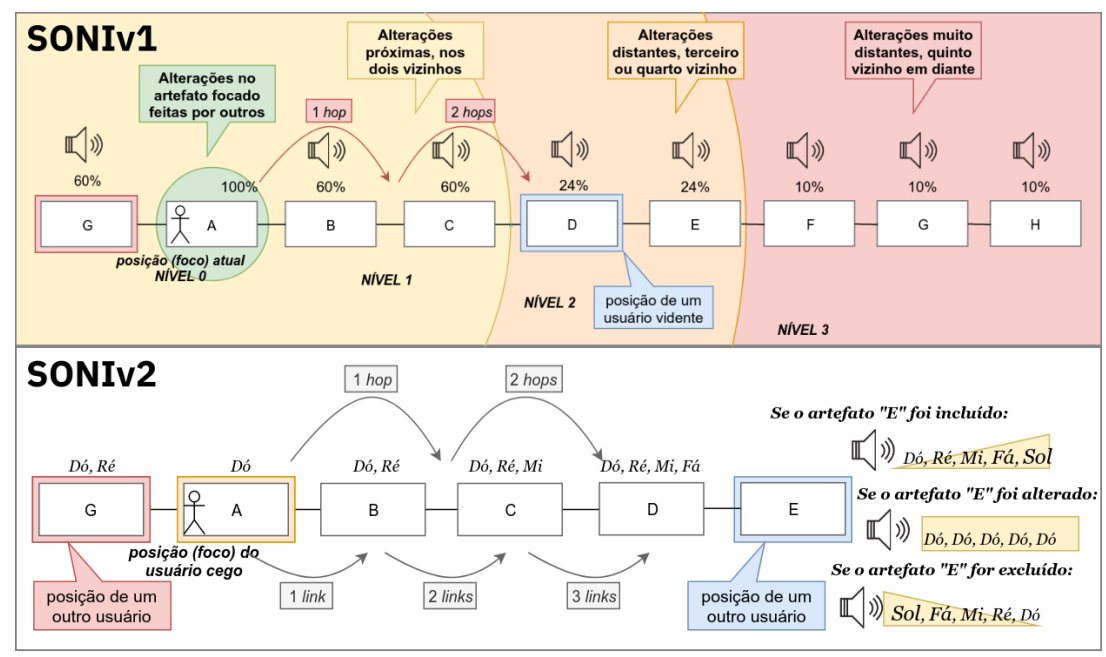

\section{Avaliação}

A ferramenta inicia a simulação com dois usuários previamente registrados, além do usuário local. O usuário participante do teste pode navegar pelos artefatos e seguir os eventos, isto é, mover-se na direção do local aonde as ações estão ocorrendo. O modelo genérico é baseado em node/links, com suporte à muitos tipos específicos de diagrama, enquanto nesta versão do protótipo os nodes são descritos concretamente como "Tabelas" e os links como "Relacionamentos". Foram conduzidas duas sessões de teste, uma para cada interface projetada, rotuladas como SONIV1, SONIv2, sobre as quais os participantes ouviram as sinalizações sonificadas que, então, eram verificadas se foram percebidas corretamente e distinguíveis por meio do teste de usabilidade, registrando a taxa de sucesso por tarefa e, ao fim da sessão, segundo as respostas às questões subjetivas do formulário NASA-RTLX (sem os pesos).

\subsection{Participantes}

Foram recrutados seis participantes para os testes, sendo três videntes, estudantes do Programa de Pós-Graduação em Computação da Universidade Federal do Rio Grande e membros do grupo de pesquisa em Informática na Educação, com experiência em diagramas e questões de usabilidade e acessibilidade, e outros três participantes cegos, alunos de cursos superiores relacionados à área da computação, um com cegueira congênita e dois com adquirida, também com conhecimento sobre informações diagramáticas.

\subsection{Preparação do Ambiente}

O ambiente de teste foi instalado nas dependências do Centro de Ciências Computacionais (C3) para o teste com videntes, nas instalações do Instituto Federal de Educação, Ciência e Tecnologia Sul-rio-grandense (IFSul) para atender um estudante cego e na a Associação Escola Louis Braille de Pelotas para atender os outros dois estudantes cegos, evitando assim que eles tivessem de se deslocar até a cidade de Rio Grande. O laptop foi posicionado de modo que os participantes videntes não observassem a interface gráfica e obtivessem apenas o retorno audível no fone de ouvido. $\mathrm{O}$ volume do áudio foi ajustado para 50\%, para não ultrapassar o limite de 50dB, sendo que 55dB é o início do estresse do 
aparelho auditivo humano [OMS 2015]. Importante: estes testes foram conduzidos antes da pandemia pelo novo coronavírus Sars-COV-2019, assim que não foram necessárias medidas de distanciamento e os experimentos tomaram lugar presencialmente.

\subsection{Procedimento}

As sessões de teste foram precedidas de um tempo para a capacitação dos participantes, de modo que eles tivessem contato com as duas interfaces, além de conhecer e experimentar as teclas de atalho. $\mathrm{O}$ modelo direcional-relativo foi apresentado visualmente aos videntes, no entanto, aos usuários cegos foi necessário apresentar por meio de um modelo físico tátil, confeccionado com EVA, corda para os links e alfinetes para rótulos em Braille, de modo que compreendessem o motivo de poder se movimentar apenas para esquerda, direita e frente, diferente de um espaço bidimensional que permitiria o movimento livre nos dois eixos. Depois da compreensão da direcionalidade por meio do material tátil, procedeu-se a sessão com a navegação audível sob o mesmo paradigma.

Figura 3. Modelo direcional tátil confeccionado em EVA para a capacitação (fonte: autor).

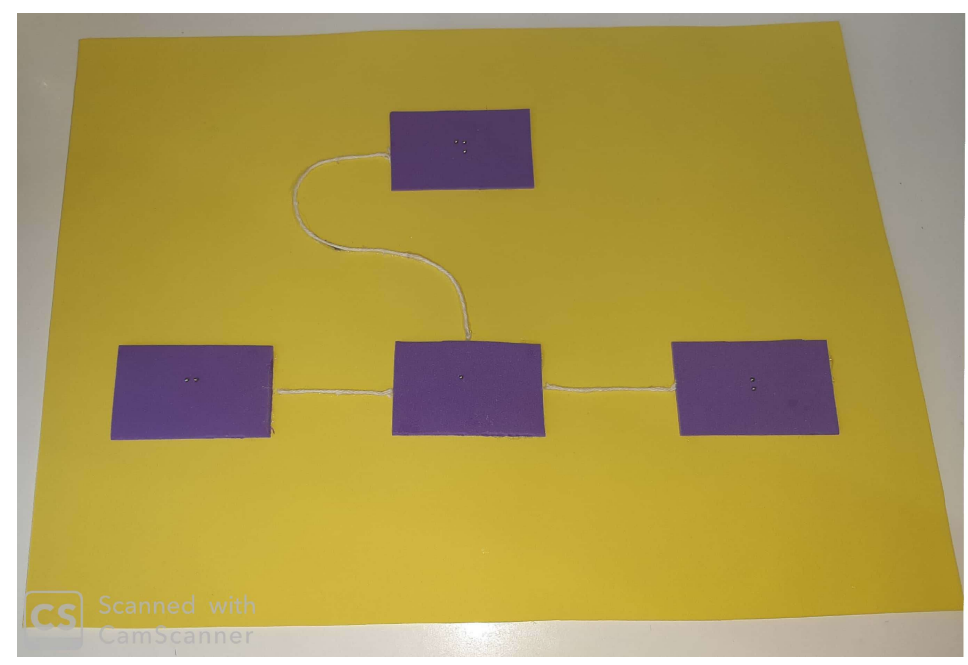

Nas sessões de teste os participantes são expostos à 12 tarefas em que o moderador, no papel de um usuário simulado, executa uma ação que dispara um saída audível, conforme interface sob teste, que comunica as quatro informações de awareness. Após cada tarefa, o participante, então, responde o que percebeu. Ao fim da sessão os usuários preenchem o formulário NASA-TLX reportando a carga subjetiva de trabalho percebida.

Os dados do teste foram armazenados em uma base de dados e organizadas pelas sessões, nas quais são armazenadas os eventos, isto é, cada atividade e sinalização reproduzida ao usuário. Os dados foram tabulados por participante e tipo de interface e os indicadores foram sintetizados e submetidos à avaliação estatística de modo a verificar a dispersão dos dados e reportar médias, valores mínimos, máximos e desvio padrão, além de um Teste $t$ pareado bicaudal para assegurar a significância na comparação das interfaces projetadas.

\section{Resultados}

As duas interfaces de usuário audíveis projetadas foram testadas por meio de interações simuladas de outros usuários as quais os participantes precisavam identificar. A quan- 
tidade de identificações corretas determina a taxa de sucesso. Ambas interfaces apresentaram taxa de sucesso a partir de $90 \%$, como pode ser visto na Figura 4 . A interface SONIv1 $(\mathrm{M}=0,9722 ; \mathrm{DP}=0,0430)$ apresentou resultados melhores que SONIv2 $(\mathrm{M}=0,9028 ; \mathrm{DP}=0,1108)$ na percepção da autoria, porém não significante $(\mathrm{t}(5)=0,19 ; \mathrm{p}>0,05)$. A interface SONIv2 obteve resultados ligeiramente melhores $(t(5)=0,70 ; p>0,05)$ que SONIv1 na identificação da ação. Quanto a noção de direção, os participantes alcançaram a taxa máxima de sucesso na interface $S O$ NIv2 (M=1, 00 ; $\mathrm{DP}=0)$, resultados que, embora melhores que SONIv1 ( $\mathrm{M}=0$, 9583; $\mathrm{DP}=0,1021$ ), não foram considerados significativos $(t(5)=0,70 ; \mathrm{p}>0,05)$. A taxa de sucesso na percepção da distância foi mais dispersa, porém as interfaces SONIv1 $(\mathrm{M}=0,8889 ; \mathrm{DP}=0,18)$ e SONIV2 $(\mathrm{M}=0,8819 ; \mathrm{DP}=0,0718)$ obtiveram resultados médios próximos.

Figura 4. Média e distribuição das taxas de sucesso por categoria de awareness (fonte: autor).

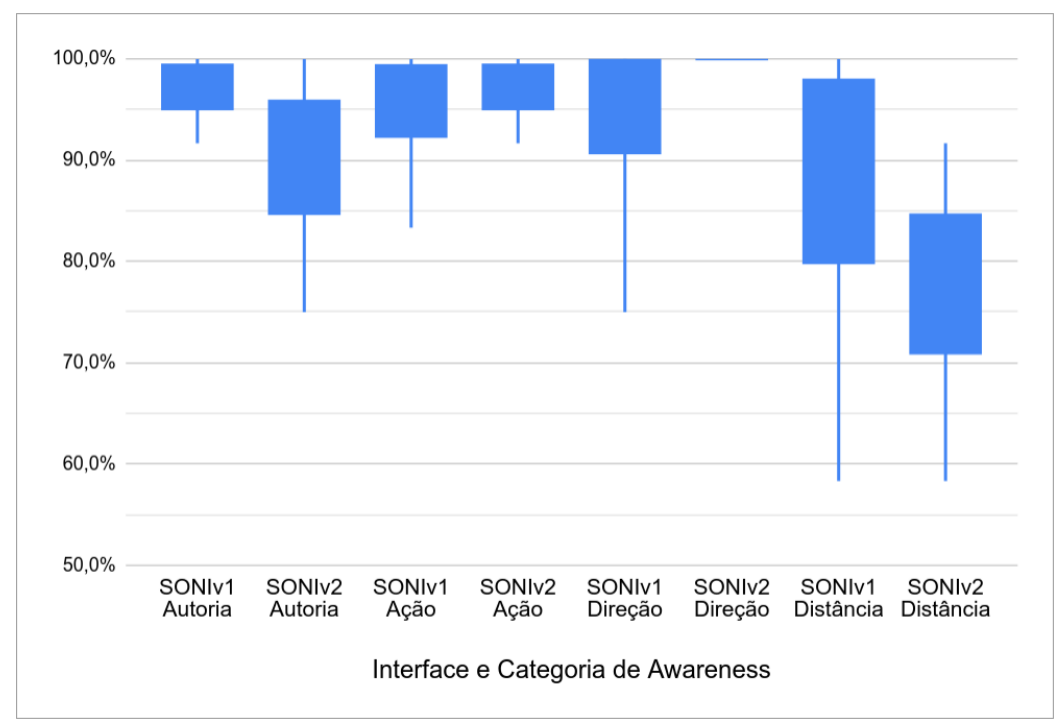

Como segundo instrumento de avaliação foi medida a carga de trabalho percebida, com respostas às seis subescalas do formulário NASA Task Load Index sem pesos. Os participantes informaram o esforço subjetivo para obter a performance objetiva descrita pela taxa de sucesso. Quanto menores forem os valores, menor a carga de trabalho percebida e, portanto, melhor o resultado. O resultado da avaliação das interfaces SONIv1 e SONIv2 pode ser visto na Figura 5.

A interface SONIv2 obteve um resultado melhor do que a SONIv1 em todas subescalas exceto frustração. O destaque é a subescala de performance, na qual os participantes relatam quão bem-sucedidos sentiram-se e sua satisfação com os resultados alcançados. Nesta métrica, a interface $\operatorname{SONIv2}(\mathrm{M}=29,17 ; \mathrm{DP}=13,93)$ foi significativamente melhor avaliada que SONIV1 ( $\mathrm{M}=15,83$; $\mathrm{DP}=14,29)$, com 99,99\% de confiança $(t(5)=0,00005 ; p<0,0001)$. Com 90\% de confiança $(t(5)=0,08$; $\mathrm{p}<0,1$ ), a carga mental percebida foi menor na SONIv2, $\mathrm{M}=22,5$; $\mathrm{DP}=11,29$ contra $\mathrm{M}=30,83 ; \mathrm{DP}=12,01$ da SONIV1. Ambas interfaces produziram resultados gerais coesos e dispersão semelhante, porém a carga de trabalho geral foi mais baixa e portanto melhor na SONIv2 com uma significância baixa $(t(5)=0,3)$. 
Figura 5. Carga de trabalho por subescala e geral das interfaces auditivas SONIv1 e SONIv2 (fonte: autor).

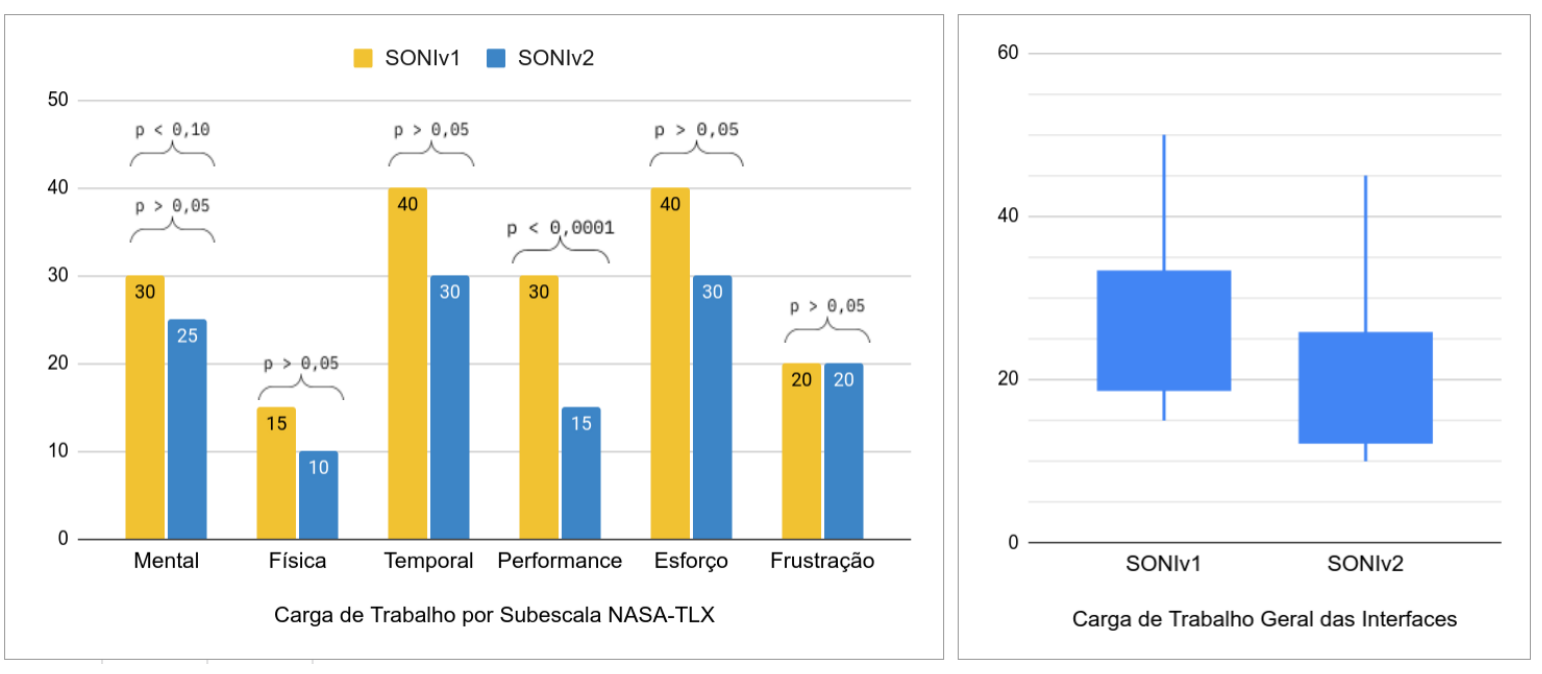

\section{Discussão e Conclusão}

Os resultados foram satisfatórios em ambas interfaces. A taxa de sucesso média foi superior a $90 \%$, exceto para a percepção da categoria "distância" com 83\%. A carga total de trabalho média ficou abaixo de 30 pontos, em uma escala até 100 . Os detalhes estão a seguir.

Embora a sonificação projetada para a percepção do agente, o awareness de " $a u$ toria/quem", tenha sido a mesma nas interfaces SONIv1 e SONIv2, os resultados foram mais expressivos para a interface $S O N I v 1$, que reproduz menos notas na sinalização, bem menos do que SONIv2, que reproduz até sete beeps. São necessários mais testes para compreender esse fenômeno, mas sabe-se de antemão que reproduções mais longas podem reduzir a performance na identificação do instrumento.

O awareness de "açãolo quê" foi melhor percebido na SONIv2. Pode ser que a reprodução de mais notas e alongadas tenham facilitado a identificação da ação, isto é, permitiram a percepção de que as mesmas estão em tom constante, ascendente ou descendente.

Os participantes perceberam com $100 \%$ de eficiência a sinalização de localização/direção na SONIv2. Estima-se que, assim como a percepção da ação, também foi influenciado pelo tempo de reprodução, ajudando na distinção do canal estéreo.

O maior desafio, desde logo, foi o de comunicar a distância. As taxas de sucesso nesta categoria foram as mais baixas e dispersas. No entanto, sabe-se que a comunicação de distância foi melhor em SONIv1. Pensa-se que o mapeamento mental, a associação do volume do áudio à distância, foi mais simples do que a contagem dos beeps exigida na interface SONIV2 com seus até sete níveis.

Finalmente, segundo as respostas do NASA-TLX, os participantes perceberam um melhor rendimento utilizando a interface SONIV2, assim como o esforço cognitivo também foi considerado menor. Uma interface com melhores resultados objetivos não necessariamente será reportada com um carga de trabalho menor. Por exemplo, a inter- 
face SONIv1 apresentou um melhor resultado na identificação da autoria e distância, no entanto, os participantes perceberam um carga de trabalho maior.

\subsection{Limitações}

As taxas de sucesso dos participantes cegos foi melhor do que a dos videntes, $99 \%$ contra $84 \%$ na interface SONIv1 e $94 \%$ contra $86 \%$ na SONIv2. Os participantes cegos também reportaram menor carga de trabalho, isto é, a percepção de menor esforço e de que as tarefas foram menos exigentes, comparados aos resultados dos videntes. Essa variação pode ter influenciado na dispersão. Além disso, alguns testes foram elencados, mas não conduzidos, como nas situações em que (a) as reproduções se sobrepõem, (b) há mais usuários presentes, (c) os coparticipantes cometem as ações, (d) ou estão online e (e) a sessão de modelagem acontece em sala de aula.

\section{Referências}

Balan, O., Moldoveanu, A., and Moldoveanu, F. (2015). Navigational audio games: an effective approach toward improving spatial contextual learning for blind people. International Journal on Disability and Human Development, 14(2):109-118.

Blattner, M. M., Sumikawa, D. A., and Greenberg, R. M. (1989). Earcons and icons: Their structure and common design principles. Human-Computer Interaction, 4(1):11-44.

Brown, A., Pettifer, S., and Stevens, R. (2004a). Evaluation of a non-visual molecule browser. In Proceedings of the ACM SIGACCESS conference on Computers and accessibility - ASSETS '04. ACM Press.

Brown, A., Stevens, R., and Pettifer, S. (2004b). Issues in the non-visual presentation of graph based diagrams. In null, pages 671-676. IEEE.

Cohen, R. F., Meacham, A., and Skaff, J. (2006). Teaching graphs to visually impaired students using an active auditory interface. In ACM SIGCSE Bulletin, volume 38, pages 279-282. ACM.

Cryer, H., Cryer, H., Home, S., Wilkins, S. M., Cliffe, E., and Rowe, S. (2013). Teaching stem subjects to blind and partially sighted students: Literature review and resources. RNIM Centre for Accessible Information.

Freire, A. P., Linhalis, F., Bianchini, S. L., Fortes, R. P., and Maria da Graça, C. P. (2010). Revealing the whiteboard to blind students: An inclusive approach to provide mediation in synchronous e-learning activities. Computers \& Education, 54(4):866-876.

Gaver, W. (1986). Auditory Icons: Using Sound in Computer Interfaces. HumanComputer Interaction, 2(2):167-177.

Godfrey, A. J. R., Murrell, P., and Sorge, V. (2018). An accessible interaction model for data visualisation in statistics. In Lecture Notes in Computer Science, pages 590-597. Springer International Publishing.

Gutwin, C. and Greenberg, S. (1996). Workspace awareness for groupware. In Conference Companion on Human Factors in Computing Systems, pages 208-209. ACM.

Gutwin, C. and Greenberg, S. (2002). A descriptive framework of workspace awareness for real-time groupware. Computer Supported Cooperative Work (CSCW), 11(34):411-446. 
Hart, S. G. (1986). Nasa task load index (tlx). volume 1.0; paper and pencil package.

Hermann, T. (2008). Taxonomy and definitions for sonification and auditory display.

INEP (2020). Censo da educação básica - 2020: Resumo técnico.

King, A., Blenkhorn, P., Crombie, D., Dijkstra, S., Evans, G., and Wood, J. (2004). Presenting uml software engineering diagrams to blind people. In International Conference on Computers for Handicapped Persons, pages 522-529. Springer.

Luque, L., Veriscimo, E., Pereira, G., and Filgueiras, L. (2014). Can we work together? on the inclusion of blind people in uml model-based tasks. In Inclusive Designing, pages 223-233. Springer.

Metatla, O., Bryan-Kinns, N., and Stockman, T. (2008). Constructing relational diagrams in audio: the multiple perspective hierarchical approach. In Proceedings of the 10th international ACM SIGACCESS conference on Computers and accessibility, pages 97104. ACM.

Miura, T., Suzuki, J., Yabu, K.-i., Ueda, K., and Ifukube, T. (2016). Diagram presentation using loudspeaker matrix for visually impaired people: Sound characteristics for their pattern recognition. In Proceedings of the 7th Augmented Human International Conference 2016, pages 1-2.

Müller, K. (2012). How to make unified modeling language diagrams accessible for blind students. In International Conference on Computers for Handicapped Persons, pages 186-190. Springer.

Nielsen, J. (2001). Success rate: the simplest usability metric. Jakob Nielsen's Alertbox, $18: 3-5$.

Nielsen, J. (2012). Why you only need to test with 5 users, 2000. Jakob Nielsen's Alertbox. Available on line from www. useit. com/alertbox/20000319. html.

OMS (2015). Make listening safe brochure.

Syal, P., Chatterji, S., and Sardana, H. K. (2016). DiGVis: A system for comprehension and creation of directed graphs for the visually challenged. Universal Access in the Information Society, 15(2):199-217.

Thapa, R. B., Ferati, M., and Giannoumis, G. A. (2017). Using non-speech sounds to increase web image accessibility for screen-reader users. In Proceedings of the 35th ACM International Conference on the Design of Communication, page 19. ACM.

Torres, M. J. R. and Barwaldt, R. (2019). Approaches for diagrams accessibility for blind people: a systematic review. In 2019 IEEE Frontiers in Education Conference (FIE), pages 1-7. IEEE.

Torres, M. J. R., Barwaldt, R., Pinho, P. C. R., de Topin, L. O. H., and Otero, T. F. (2020). An auditory interface to workspace awareness elements accessible for the blind in diagrams' collaborative modeling. In 2020 IEEE Frontiers in Education Conference (FIE), pages $1-7$.

Turner, C. W., Lewis, J. R., and Nielsen, J. (2006). Determining usability test sample size. International encyclopedia of ergonomics and human factors, 3(2):3084-3088. 